\title{
Morphofunctional features and physical fitness of boys 6 - 9 years old under the influence of various physical activity
}

\author{
Tamara Abramova ", Tatyana Nikitina, Anastasia Polfuntikova, Kristina Oblog, and Faina \\ Iordanskaya \\ Federal Science Center for Physical Culture and Sport, 105005, Moscow, Russia
}

\begin{abstract}
The aim of this research was to investigate morphofunctional features and physical fitness of boys 6 - 9 years old under the influence of various physical activity. The research performed in 2020 comprised 246 boys of $6-9$ years old with different physical activity (121 children who are not involved in sports; 79 martial artists and 46 gymnasts with experience of 8 months and 4 years old). It was found that systematic sports activities of 6 - 9 years old boys have a positive effect on physical fitness and optimize morphofunctional indicators of physical development. The effect of the sport on the physical development and fitness of the boys depends on the start date and time of training sessions: a higher level of young gymnasts physical fitness is accompanied by a more intense adaptation of the cardiovascular system relative to martial artists. Differences in morphofunctional state and physical fitness between boys who are not involved in sports and young athletes are least significant in 6 year old boys, intensifying as growing up and increasing sport experience. It is more typical for gymnasts.
\end{abstract}

\section{Introduction}

The formation of physical fitness in ontogenesis occurs in accordance with the development of physique and functionality under the influence of heredity and external influences, including motor activity [1].

The variety of organized children's physical activity 6-10 years old is determined by two sides: for the majority of children it is the regulation of physical education in preschool and primary school. Here physical education classes are limited by 2 times a week; for a smaller part of children it is the regulation of sports. It is defined by federal standards of sport training as 6 hours of training work a week [2]. Timing (volume) differences are supplemented by the content ones with physical education oriented on general physical training with sports activities focus on special skills development from the very beginning of training. It is dictated by the need to master optimal technique, including injury prevention [3].

*Corresponding author: atf52@bk.ru 
In spite of longstanding discussion about advantages and disadvantages of early entry into sport for the present it is evident that sport activities at the age of 6 to 10 form more high physical fitness level optimizing also the physical development. However, it is also obviously, that sports at the tender age are already focused on specific development not only of physical fitness components, but also of the various of morphofunctional indicators $[4,5]$. Single combats and gymnastics are recognized as common sports with early involvement of children. Single combat athletes at the age of 6 exercise 2 times a week for 45 minutes, which increase to 60 minutes from 7 years old and to 90 minutes and $2-3$ times a week since 8 years and older. Gymnastics at the age of 6 is oriented on 90 minutes 3 times a week, at the age of 7 - on 4 hours 5 times a week, further - on $4-5$ hours 6 times a week.

The aim of this research is to examine the impact of sports practice on the morphofunctional features and physical fitness of 6 - 9 years old boys, involved into single combats and gymnastics.

\section{Materials and methods}

The survey included methods recommended for assessing the physical development and fitness of children and GTO standards [3, 6].

Methods: anthropometry, caliperometry, physiometry, heart rate, pedagogical, graphic analysis [7, 8, 9]. Were determined: length and body weight, arm and leg circumference measurements, fat deposition, lung capacity (exhale), heart rate monitoring, hand dynamometry, shuttle run time (10m x 3), length of long jump with both feet, flexibility test in leaning forward from a standing position on gym bench, pushups (in 60 seconds) and curl-up test (30 seconds). The graphical analysis used a geometric representation of the trajectories of a dynamic system in a phase plane that combines two variables of the same system - the independent variable and its rate of change. It reflects the general laws of the system's behavior $[9,10,11]$.

The participants in this study numbered 246 boys aged $6-9$ years with various physical activity (121 non-athletes boys (NA - control groups); 79 single combat athletes and 46 gymnasts with sport experience from 8 months to 4 years). The number of participants in every age group: non-athletes - 25 - 39 boys; gymnasts - 10 to 12 boys, single combat athletes - 20 to 29 boys. The testing was conducted with informed consent of the parents.

\section{Results and discussing}

Growing up changing of morphofunctional status and physical fitness of children aged 6 10 years among them there are non-athletes, young gymnasts and single combat athletes is shown on figures $1-4$.

Total body size, including length and body weight, chest girth as main markers of child developing in these sports beginning from the age of 6 until 9 reflect biological growth patterns with elements of influence requirements of these types of sport. This manifests in complete identity of development level and pace in single combat athletes and non-athletes boys (NA) (Fig. 1). In contrast, gymnastics demonstrate concern to select children with lower (within norms) length and body weight than control group. It is accompanied by similar level and pace of body mass increasing with control group and single combat athletes, evidencing balanced development of young gymnasts. Chest girth in growing up is identical in all groups, in spite of length and body weight. It indirectly points out relatively more developed torax in gymnasts. 


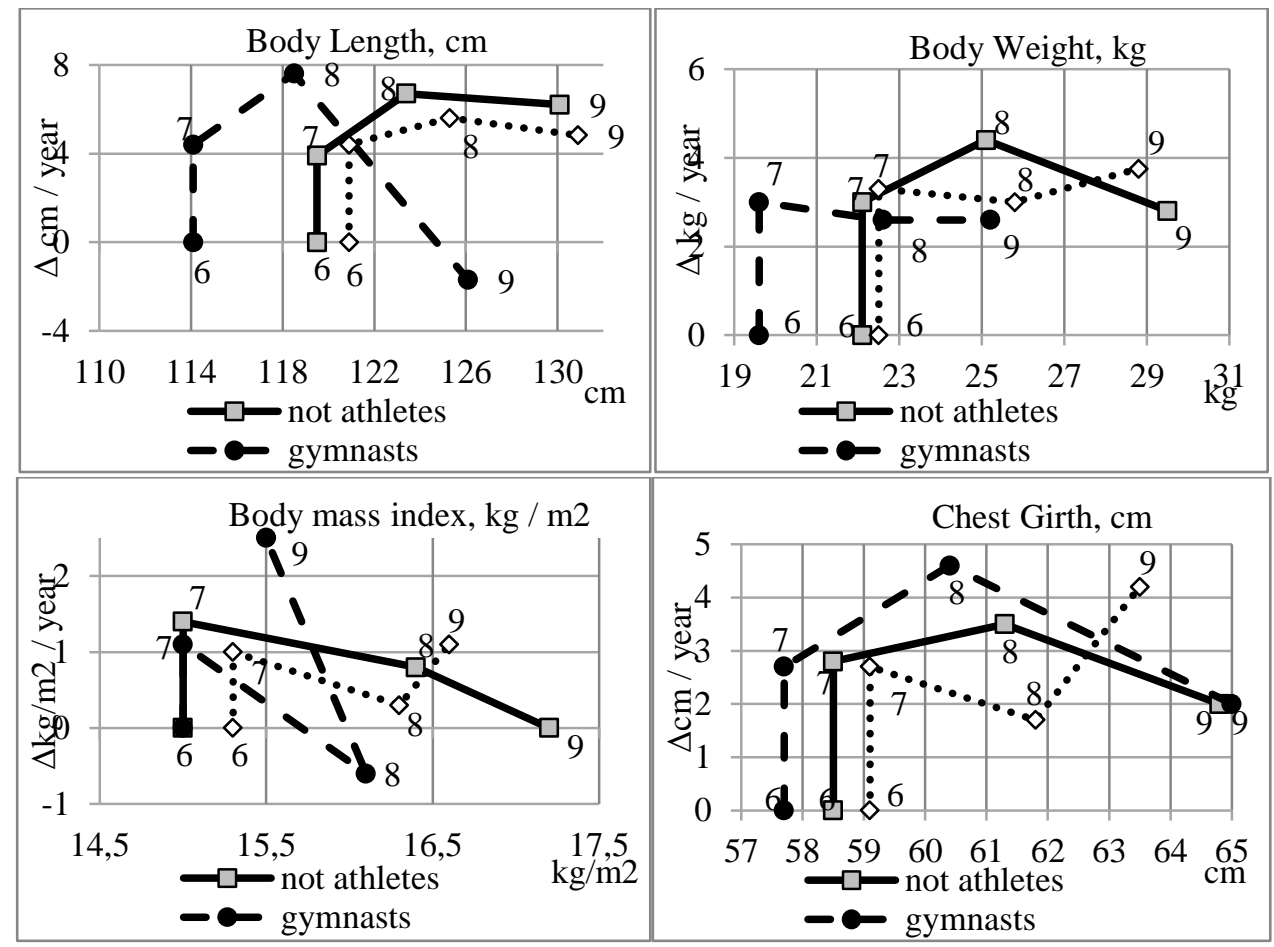

Fig. 1. Growing up dynamic of length, body weight, body mass index and chest girth in $6-9$ aged non-athletes, single combat athletes and gymnasts ( $y$-axis - changing in year, $x$-axis - rate of indicate; point numbers match the age)

Development of labile body mass components from age of 6 is objective marker of systematic sport activities influence (Fig. 2). During the age of 6 to 9 it manifests in more and practically equal increasing of muscles mass in young athletes groups (from 43,7 - 44,6 to $45,8-46,4 \%$ ) in relation to non-athletes children (from 41,2 to $42,0 \%$ ) and also less pace and level of fat mass increasing concerning to not involved in sport peers (from 16,5 to $23,0 \%$ ), which is typically for gymnasts in greater extent (from 9,5 to 9,3\%) and less for single combat athletes (from 12,6 to $14,8 \%$ ).
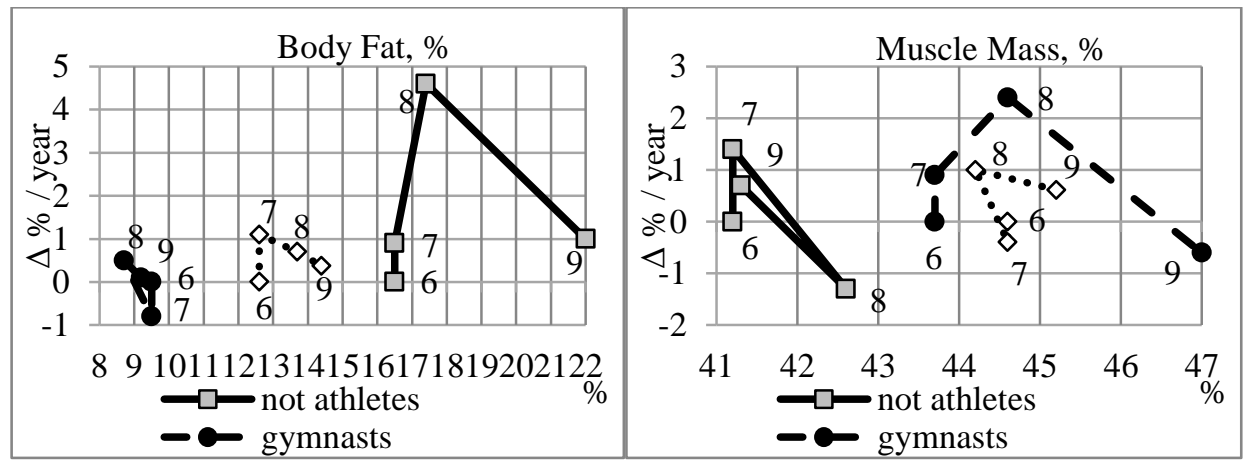

Fig. 2. Growing up dynamic of muscles and fat in $6-9$ aged boys non-athletes, single combat athletes and gymnasts ( $\mathrm{y}$-axis - changing in year, $\mathrm{x}$-axis - rate of indicate; point numbers match the age) 
The analysis of physical fitness indicators confirms higher level in young athletes group and among them - in young gymnasts group (Fig. 3). Age ranges of physical fitness indicators changes in groups vary widely in average values.

Absolute hand strength is the highest in all ages of athletes group, equate increasing since 6 till 9 years from 7,5 to $14-15 \mathrm{~kg}$. It is meaningfully higher than in non-athletes group of boys (from 6,8 to $10,7 \mathrm{~kg}$ ) with equal improving during three years in young athletes group $(84-87 \%)$, whereas in group of not involved in sports boys hand strength raises on $57,5 \%$.

Hand strength in relative terms is the highest in all aged groups of gymnasts, increasing since 6 till 9 years from 38 to $50 \%$. It is meaningfully higher than in single combat athletes group (from 34 to $43 \%$ ) with equal improving during three years in young athletes groups (28-31\%), while in non-athletes group of boys hand strength is reliably lower and during this time it is increasing from 30 to $34 \%$ (on $9,4 \%$ ).

The results of long jump, which reflected development of legs explosive strength and coordination, are the highest in all ages also in gymnasts group, increasing since 6 till 9 in average from 147 to $182 \mathrm{~cm}$, which is significantly higher than in single combat athletes group (from 117 to $153 \mathrm{~cm}$ ), whereas in group of not involved in sport boys the length of jump is reliably less and during this time it is improving from 107 to $137 \mathrm{~cm}$, what provides practically equal raising test results in three years within the limits of $23-28 \%$.

Time of shuttle running as indicator of agility developing shows the best results also in group of gymnasts of all ages, decreasing since 6 till 9 years from 9,8 to 8,1 sec, which is noticeably better than in group of single combat athletes (from 11,1 to 9,2 sec), while among non-athelets boys the dynamic of this indicator changes within the interval from 11,4 to $9,7 \mathrm{sec}$, with common improving in three years in all groups $(17 \%)$.

The flexibility initially is the best and high in group of gymnasts, increasing since 6 till 9 years from 13,6 cm to $17,0 \mathrm{~cm}$ (on 25\%), it is average for age groups in single combat athletes, decreasing in three years on $52 \%$ (from $4,8 \mathrm{~cm}$ at the age of 6 to $2,3 \mathrm{~cm}$ at 9), and it is low for non-athletes boys improving since 6 till 9 years from -1 to $1 \mathrm{~cm}$.

In spite of physical fitness superiority of young athletes group the least differences with not involved in sport group of children are marked at the age of 6 , in average accounting for $7,9 \%$ for single combat athletes, increasing at $7-9$ year till $16-19 \%$; for gymnasts $21,7 \%$ raising till $30-37 \%$ during 7 to 9 years.

The provided data demonstrate the most express gymnastics positive impact on physical fitness development components in $6-9$ years old children. It is correlated with the least fat deposing and increased growth of muscle mass in spite of lower within age norms and balanced length and body weight.

Changes of morphofunctional indicators and physical fitness in single combat athletes in the same age interval are also positive, but less express. With high probability differences in effects are determined by early start and more training volume (timing) in gymnastics comparing with single combats. In this context the analysis of functional indicators dynamic in cardiovascular system and autonomic nervous system as leading functional system of life support represents as important (Fig. 4).

Heart volume's and vascular lumen's age-related heterochrony of development with relatively slower heart volume increase, while arteries and capillaries lumen is relatively larger than in adults against reduced stroke volume of blood forms reduced blood pressure, it is compensated by a higher heart rate $[10,11]$. Increasing in growing up blood pressure is the most intensive in children at the age of $2-3$ years and in pubertal development with parallel heart rate reducing $[12,13]$. 


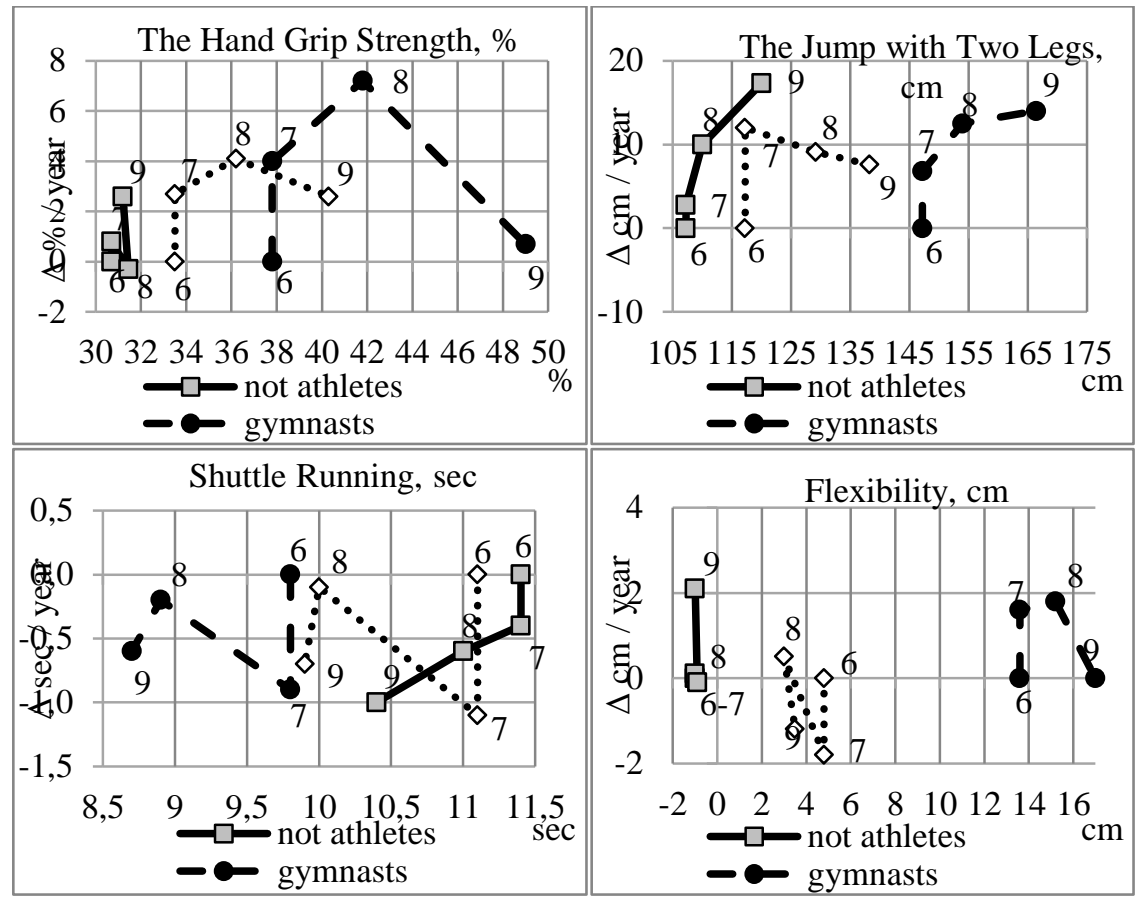

Fig. 3. Growing up dynamic of testing results of hand grip strength, jump with two legs, shuttle running and flexibility in $6-9$ aged boys non-athletes, single combat athletes and gymnasts (y-axis changing in year, $\mathrm{x}$-axis - rate of indicate; point numbers match the age)

Cardiovascular's and autonomic nervous system's functional conditions indicators agerelated dynamic in young single combat athletes during $6-9$ years is characterized of blood pressure increasing in average from 101/66 to $114 / 75 \mathrm{~mm} \mathrm{Hg}$ (upper limit of the norm), which forms pulse pressure in interval $35-39 \mathrm{~mm} \mathrm{Hg}$ (normal) with practical permanence of pulse (91 bpm at 6 year old and 90,2 bpm at 9). Timing adaptation of cardiovascular system in 6 to 9 years period in gymnastics is manifested by systolic blood pressure raising with directed reduction of diastolic blood pressure (from 100/68 to 114/42 $\mathrm{mm} \mathrm{Hg}$ ) and respective increasing of pulse pressure from 32 to $52 \mathrm{~mm} \mathrm{Hg}$, it is accompanied by directed reduce of pulse from increased at 6 years $(106 \mathrm{bpm})$ to normal at 8 years $(90 \mathrm{bpm})$ with raising to higher $(98 \mathrm{bpm})$ at 9 years.

The comparison of young athletes group with control group reveals basic distinctions related to trend of age increasing systolic blood pressure in athletes groups, which accompanied of higher diastolic blood pressure forming in single combat athletes group, in gymnasts group - expressed reducing of diastolic blood pressure. It expresses by pulse pressure raising and high heart rate. It cumulatively reflects the work high in volume or (and) in intensity showing the effect of specializing on cardiovascular system, summarily in any event causing tense reaction of heart. It is more extent shown in gymnastics.

Differences in forming of cardiovascular and autonomic nervous system regulation are the least significant at the age of 6 , intensifying as growing up and sport experience. However even at the age of 6 considerably higher pulse and diastolic blood pressure in group of gymnasts are drown the attention, reflected higher tense in heart function. 


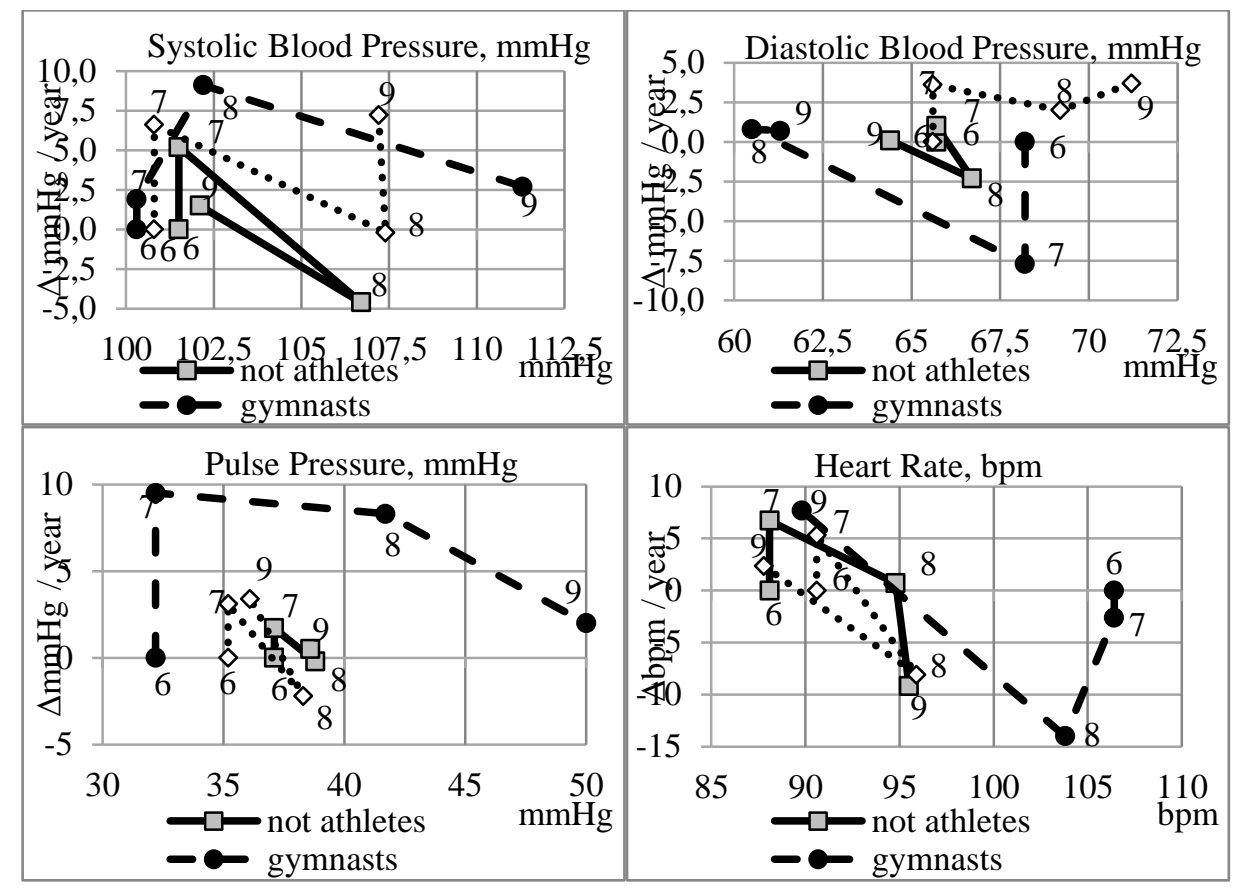

Fig. 4. Growing up dynamic of blood pressure, pulse pressure and heart rate in $6-9$ aged boys nonathletes, single combat athletes and gymnasts (y-axis - changing in year, x-axis - rate of indicate; point numbers match the age)

The provided results correspond with data received in research about volume of physical activity at school, evidencing that children of $6-9$ years old exercised 4 times a week for 45 minutes compare to children exercised 2 times a week 45 minutes in physical education are different in higher indicators of physical fitness [13]. Studying more than 16000 of children in Europe aged 2 - 9 revealed positive impact of physical activity on reducing risk of cardiovascular system disorders with the recommendations to exercise no less than 60-85 minutes a day [14].

The summarized results of 24 researches published in journals of Pubmed and SportDiscus data bases are significant. They found the osteogenic effect of gymnastics manifesting activation forming mineral bone density in growth process in spite of diet restrictions, hormonal disorders and reduced fat deposing [15]. Received earlier own data about young football players and cyclists physical development and physical fitness confirm also positive impact of sport exercises at the age of $6-10$ on development and physical fitness, but also evidence about peculiarities of cardiovascular system formation autonomic regulation under the effect of special exercises directed on specific for sport type skills improving $[16,17]$.

\section{Conclusion}

The performed research evidently shows that systematic sport exercises of $6-9$ years old boys influence positively on physical fitness and optimize morphofunctional indicators of physical development. Sport type's impact on boys physical development and physical fitness mostly depends on term of start and timing (volume) of training exercise. It reflects higher level of young gymnasts physical fitness and accompanies by more pronounced manifestation of cardiovascular system tense adaptation compare to single combat athletes. 
Differences in rate of morphofunctional indicators and physical fitness between nonathletes boys and young athletes the least significant at the age of 6 , intensifying by growing up and sport experience. It is more typical for gymnasts. Sport exercises of 6-9 years old boys require obligatory medico-biological control.

\section{References}

1. V.K. Balsevich, Human ontokinesiology (Theory and practice of physical culture, Moscow, 2000).

2. Federal standards of sport, URL: https://www.minsport.gov.ru/sport/podgotovka/82/5502/ (date of request - 07.02.2020).

3. V.I. Lyakh, A.A. Zdanevich, The complex program of physical education of 1-11 grades students (Enlightenment, Moscow, 2004)

4. T.F. Abramova, T.M. Nikitina, A.V. Polfuntikova, Bulletin of the Moscow University, Series 23, The Anthropology, 3, 5-14, (2019)

5. E.E. Khvatskaya, N.E. Latysheva, Bulletin of Pskov state University, Series «Psychological and pedagogical Sciences», 1, 194-200, (2015)

6. The order of Russian Ministry of sports of 12.02.2019 № 90, [website] URL: https://minjust.consultant.ru/documents/ 42248?items $=1 \&$ page $=5$ (date of request 05.02.2020)

7. T.F. Abramova, T.M. Nikitina, N.I. Kochetkova, Labile components of body weightcriteria for General physical fitness and control of current and long-term adaptation to training loads. (Skyprint, Moscow, 2013)

8. G.N. Serdyukovskaya, Organization of medical control over the development and health of preschoolers and schoolchildren on the basis of mass screening tests and their improvement in the conditions of kindergarten, school (Moscow, 1995)

9. Yu.M. Romanovsky, N.V. Stepanova, D.S. Chernavsky, What is mathematical Biophysics. (Enlightenment, Moscow, 1971)

10. V.A. Melnik, Health and environmental issues, 3 (41), 116-121, (2014)

11. D.B. Dorjieva, I.A. Badmayeva, T.N. Erneva, Natural Sciences and medicine: theory and practice (Novosibirsk, 2018)

12. I.N. Usov, Healthy child: a guide for the pediatrician (Minsk, Belarus, 1994)

13. A.-C. Sollerhed, G. Ejlertsson, Scand J Med Sci Sports, 18, 102-107, (2008)

14. L.B. Andersen, M. Harro, L.B. Sardinha, K. Froberg, U. Ekelund, S. Brage, S.A. Anderssen, The European Youth Heart Study, Lancet, 368, 299-304 (2006)

15. Jaak Jürimäe, Rita Gruodyte-Raciene, Adam D.G. Baxter-Jones, J Sports Sci Med, 17(2), 245-258, (2018)

16. T.F. Abramova, T.M. Nikitina, A.V. Polfuntikova, Theory and practice of physical culture, 2, 60-62, (2020)

17. E.A. Raspopova, Y.A. Postolnik, Pedagogico-psycological and Medico-biological Problems of Physical Culture and Sport, 11(1), 57-63 (2016) 